\title{
An Improved Silver Ion HPLC Combined with Capillary Gas Chromatography of cis/trans Fatty Acids in Alimentary Fats and Human Milk Fat
}

\author{
Andrzej Stolyhwo • Jarosława Rutkowska
}

Received: 2 January 2012 / Accepted: 4 June 2012 / Published online: 19 June 2012

(C) The Author(s) 2012. This article is published with open access at Springerlink.com

\begin{abstract}
A technique of analysis is described using a silver ion-loaded HPLC $\left(\mathrm{Ag}^{+} / \mathrm{HPLC}\right)$ column, gradient elution, and a laser light scattering detector followed by capillary gas chromatography (GC) for the determination of the fatty acid methyl ester (FAME) composition of rapeseed oil, the respective hydrogenated fat, some dietary fats, and human milk fat. With the use of $\mathrm{Ag}^{+} / \mathrm{HPLC}$, FAMEs were separated into the following classes: saturated, trans-monounsaturated, cis-monounsaturated, trans-trans-di-unsaturated, trans-cisdi-unsaturated, $c i s-c i s-d i-u n s a t u r a t e d$, and higher unsaturated FAMEs. Within each class, partial separation of positional isomers was also possible. Fractions of the eluate containing trans and cis $\mathrm{C} 18: 1$ were separately collected and re-analyzed by GC in order to quantitate the positional isomers eight trans and nine cis in rapeseed oil and in human milk fat. The profiles of eight trans positional isomers in human milk fat and in hydrogenated rapeseed oil were alike but entirely different from that of six C18:1 trans isomers in bovine milk fat. Out of nine $\mathrm{C} 18: 1 \mathrm{cis}$ isomers found in hydrogenated fat, only $\mathrm{C} 18: 1-9 c$ and $\mathrm{C} 18: 1-11 c$ were found in both human and bovine milk fat which may indicate some discrimination which occurs for certain isomers in fatty acid metabolism.
\end{abstract}

\footnotetext{
A. Stolyhwo

Department of Dietetics, University of Bydgoszcz,

Unii Lubelskiej St. 4c,

85-059 Bydgoszcz, Poland

J. Rutkowska ( $\square)$

Department of Instrumental Analysis, Faculty of Human Nutrition and Consumer Sciences, Warsaw University of Life Sciences, Nowoursynowska St. 159c,

02-776 Warsaw, Poland

e-mail: jaroslawa_rutkowska@sggw.pl
}

Keywords trans fatty acids · Gas chromatography · Silver ion-loaded HPLC · Laser light scattering detector . Cold-pressed rapeseed oil $\cdot$ Hydrogenated fats $\cdot$ Human milk

\section{Introduction}

The Codex Alimentarius Commission 2004 has adopted a definition proposed by the Danish delegations which is "trans fatty acids are defined as all the geometrical isomers of monounsaturated and polyunsaturated fatty acids (FAs) having nonconjugated (interrupted by at least one methylene group $\left(-\mathrm{CH}_{2}-\mathrm{CH}_{2}-\right)$ carbon-carbon double bonds in the trans configuration". This includes the trans monoenes (mainly steroisomers of elaidic acid) and the trans isomers of polyunsaturated FAs (e.g., trans dienes, trans trienes etc.) with non-conjugated carbon-carbon double bonds, produced through hydrogenation of oils and fats (both vegetable and animal/marine origin) in the presence of a suitable chemical catalyst. The definition, however, excludes conjugated trans FAs present naturally in animal fats and their products that include conjugated linoleic acid. The US FDA defined trans FA as "unsaturated FAs that contain one or more isolated (i.e., nonconjugated) double bonds in a trans configuration (Handa et al. 2010; USFDA 2010). In 2004, Denmark was the first country that introduced a limitation on the content of industrially produced trans FAs in foods. USA and Canada were the first two countries to introduce the mandatory declaration of trans FAs (CFIA 2008; USFDA 2010).

Following the abovementioned regulations, industry practices regarding unsaturated fat hydrogenation changed over last 10 years. In practice, several temperature points exist near which critical effects in the reaction course are to be expected. For example, a low-temperature $\left(120{ }^{\circ} \mathrm{C}\right)$ 
hydrogenation may result in less than $20 \%$ of trans FA. However, the so obtained fats would not meet the requirements of baking, confectionery, and frying processes. Fats for baking require a long plastic range. One of the approaches is the so-called trans-suppressive hydrogenation in which the melting point is being approached gradually and, in consequence, the solid fat content or solid fat index curves are flatter than normal as required of a shortening or of baking fats. In that approach, a fresh or nearly fresh nickel catalyst is used at the usual nickel/oil level $(0.05-0.15 \%)$, the pressure of up to 5 at and temperature up to $160{ }^{\circ} \mathrm{C}$. In case the texture of that fat does not meet the requirements, fully hardened vegetable oil $(3-5 \%)$ is being added. On the other hand, hydrogenation above $160{ }^{\circ} \mathrm{C}$ renders best functional properties of confectionery fats due to an increased content of trans-isomers (Patterson 2009).

The principal structural changes of hydrogenated fats are as follows:

- Structural (positional) isomerization of FAs which involve the migration of double bonds from the most abundant $\Delta-9$ or $\Delta-11$ positions along the hydrocarbon chain of unsaturated FA

- Geometrical isomerization in which the cis isomers of FAs (exclusively present in plant oils) are converted during hydrogenation to their trans analogs. The process is associated with structural (positional) isomerization

\section{Physiological Consequences of trans-Fatty Acids Intake}

The use of hydrogenated fats for human consumption provokes controversial discussions, between nutritionists and representatives of the fat industry, regarding the health effects of trans-isomers of unsaturated FAs (Wolf 1995; Lichtenstein 1993; Applewhite 1993; Haumann 1994; Mensink and Katan 1990; Willet et al. 1993; Judd et al. 1994; Lopez-Garcia et al. 2005), one of the effects being a negative impact on prostacyclin synthesis. Namely, trans FAs contained in hydrogenated fats cannot serve as precursors for prostacyclin synthesis and, additionally, inhibit that synthesis. Moreover, trans FAs get incorporated into the membrane lipids of blood vessels and muscles, thus displacing the essential linoleic, linolenic, and arachidonic acids (Kummerow et al. 2004).

At present, partially hydrogenated fats are one of the main components of different spreads, margarine blends, creamers, etc. They are also hidden in considerable quantities in bakery products, French fries, and other commercial products. Consequently, their occurrence may be expected in human structural lipids. trans FA isomers detected in human milk (Wolff 1995; Chen et al. 1995; Picciano and Perkins 1977; Koletzko et al. 1988); Craig-Schmidt et al. (1984) reported a linear correlation between the trans isomer contents in human milk and in dietary fat, the content of trans FA isomers in human milk ranging from 1.3 to $7 \%$ (Chen et al. 1995).

The significance of an early intake of trans FAs was reviewed by Larqué et al. (2001). Infant tissues incorporate trans FAs from maternal milk thus raising the level of linoleic acid and relatively decreasing those of arachidonic and docosahexaenoic acids. This suggests an inhibitory effect of trans FAs on liver $\Delta-6$ fatty-acid desaturase activity. In contrast to blood and liver, the brain appears to be protected from the trans fatty acid accumulation in experimental animals but no data have yet been reported for human newborns. Further investigations in human beings are needed to definitively establish the potential physiological consequences of trans FA intake during the neonatal period (Larque et al. 2001).

\section{Determination of trans-Fatty Acids in Alimentary Fats}

In order to determine the composition of hydrogenated fats and their suitability for human consumption, the analytical methods used should be capable of determining all changes in the molecular structures of FAs and triacylglycerols, the products of industrial hydrogenation of plant oils. It ought to be emphasized that the official AOAC methods 985.21 or 965.35 are not selective or precise enough for characterizing structural changes in hydrogenated fats (AOAC 1990a, b).

Delmonte and Rader (2007) focused on some critical aspects of trans FA quantitation by current methodologies described in AOAC Official Method 996.06 and AOCS method Ce 1h-05. The cited authors concluded that in as much those methods were based on $100 \mathrm{~m}$ highly polar capillary columns and, preferably, hydrogen as a carrier, several FAs belonging to different classes (i.e., saturated, trans FAs, and mono- and polyunsaturated) are still not adequately separated. Fractionation by liquid chromatography prior to gas chromatography (GC) analysis can eliminate the co-elution of FAs belonging to different classes but incorrect practices might lead to greater analytical errors than those caused by those co-elution procedures that affect direct GC analysis (AOCS 2005; AOAC 2005; Delmonte and Rader 2007). Some modifications of the abovementioned standard GC methods that enable separation of C18:1 12- $t$ and C18:1 13- $t$ isomers occurring in significant levels but often remaining unresolved from the C18:1 9-cis peak, were proposed by Phillips et al. (2010).

Ratnayake and Beare-Rogers (1990) showed that in gasliquid chromatography (GLC) even the use of a long fused silica column $(60 \mathrm{~m} \times 0.25 \mathrm{~mm}$ ID) coated with a $50-\mu \mathrm{m}$ film of SP-2340 (biscyanopropylphenylpolysiloxane) is not 
sufficient for an adequate separation of positional and geometric isomers of monounsaturated FA in margarines containing hydrogenated fats. As a remedy, more selective separations of FA isomers became possible with the use of argentation chromatography, in particular with the introduction of stable ion-exchange HPLC columns loaded with silver ions (Christie 1987; Stefanow et al. 1988; Christie et al. 1988). Christie and Breckenridge (1989) successfully used such columns for the separation of fenacyl derivatives of geometrical and positional (partial separation) isomers of FAs, as well as for a complete separation of the molecular species of triacylglycerols with different stereospecific structures (Christie 1988, 1991). Application of silver-loaded columns requires, however, the use of a gradient elution technique which creates problems in detection of the separated FAs or triacylglycerols. A prefractionation of cis and trans FAs could be performed by silver-ion thin-layer chromatography. Destaillats et al. (2007) compared that technique with the preseparation by RP-HPLC (according to Juanéda 2002), direct quantification by gas-liquid chromatography and with the estimation by applying triacyloglycerols formulas to milk fat. They reported that accurate measurement of total trans C18:1 and of vaccenic acid, the principal trans C18:1 isomer in milk fat, can be performed by direct GLC under optimum chromatographic conditions (Golay et al. 2006). The analysis of isomeric distribution of trans $\mathrm{C} 18: 1$ could not be achieved by direct GLC analysis since a fractionation step was required. RP-HPLC fractionation does not allow minor trans C18:1 isomers, such as trans 4/5 C18:1 isomers, to be isolated (Destaillats et al. 2007).

Lipids, in general, are known not to absorb UV radiation over $220 \mathrm{~nm}$. In contrast, solvents that could preferentially be used for HPLC-gradient elution of fatty acid methyl ester (FAME) and triacyloglycerols are not UV-transparent below $220 \mathrm{~nm}$. The laser light scattering detector (LLSD) described earlier (Stolyhwo et al. 1983, 1984, 1985, 1987) with several recent technical modifications, appears to be a very useful detecting device for use with both silver-loaded or RP columns in gradient elution HPLC. The merits of LLSD detectors were emphasized in the AOCS Ce $1 \mathrm{~g}$ 96 instruction for detecting trans-isomers by silver ionexchange HPLC. In this study, a split-flow device was placed before the detector and fraction collection was monitored by the detector which enabled collecting peaks free of impurities (AOCS 2009).

The aim of the study was to adapt the methods of capillary gas chromatography and silver ion chromatography combined with the modified laser light scattering detector $\left(\mathrm{Ag}^{+} / \mathrm{HPLC} /\right.$ LLSD) for the determination of structural changes of fatty acids in hydrogenated fats and for monitoring the presence and content of structural and geometric isomers of FAs in dietary fats and in human milk.

\section{Material and Methods}

Samples and Reagents

Virgin rapeseed oil was obtained in the laboratory by cold-pressing fresh rapeseeds in a stainless steel, laboratoryscale hydraulic press. Samples of palm oil were obtained from the Institute de Corp Gras (Pessac, France). Samples of hydrogenated rapeseed oil and fat for baking came from the Olivit factory (Gdansk, Poland). Samples of stick margarine were purchased at a grocery store. French fries were purchased at a fast food restaurant in Warsaw (Poland). Samples of human milk were collected under controlled conditions and kindly donated by one of our friends who nourished her baby. Samples of bovine milk originated from a farm located in central Poland.

Acetonitrile, hexane, and dichloromethane were LiChrosolv grade (E. Merck, Darmstadt, Germany). Acetone and 2-propanol (AR grade) were obtained from Polish Chemical Reagents (Gliwice, Poland). Before use, the latter two solvents were filtered using a $0.2-\mu \mathrm{m}$ filter and distilled. Before and during analyses all solvents were sparked with helium. FAMEs and their cis/trans isomer standards were purchased from Sigma Aldrich (Poland).

\section{Fat Extraction}

Fat was extracted from milk samples according to the method of Rose-Gottlieb (AOAC method no. 905.02) (AOAC 1990c). Crushed French fries were dried and then extracted in a Soxhlet extractor with hexane-methylene chloride 50/50 v/v. After filtration, the solvents were evaporated under vacuum. FAMEs were prepared by saponification of samples with $0.5 \mathrm{~N}$ sodium hydroxide in methanol. The resulting soaps were converted directly to the FAME with $14 \% \mathrm{BF}_{3}$ solution in methanol according to AOAC Method 969.33 (AOAC 1990d). Isomerization of rapeseed oil was carried out by the use of nitrous oxides. A diluted solution of sodium nitrite was vigorously shaken with the oil followed by dropwise addition of diluted nitric acid while shaking.

\section{Instrumentation and Procedures}

GC analyses were carried out on a Hewlett-Packard Model 6890 GC using split-injection (120:1), flame ionization detector, and Rtx 2330 column (Restek Corp. Bellefonte, PA; $105 \mathrm{~m} \times 0.25 \mathrm{~mm}$ filled with fused silica and containing $0.20 \mu \mathrm{m}$ film of biscyanopropylphenylpolysiloxane). The initial column temperature $\left(150{ }^{\circ} \mathrm{C}\right)$ was kept for $25 \mathrm{~min}$, then increased at a rate of $2{ }^{\circ} \mathrm{C} / \mathrm{min}$ up to $205^{\circ} \mathrm{C}$; FID range was $16 \mathrm{pA}$.

HPLC analyses for the separation of FAME isomers utilized a silver-loaded column (ChromSpher 5 Lipids, $250 \times 4.6 \mathrm{~mm}$ ID, Chrompack, Middelburg, the Netherlands). 
The following solvents were used: hexane-methylene chloride 90/10 $v / v$, methylene chloride/acetone 10/90 $v / v$, and acetone and acetone/acetonitrile 20/80. Gradient elution: $t(\mathrm{~min})=0$, $100 \% \mathrm{~A} ; t=8.0,88 \% \mathrm{~A} / 12 \% \mathrm{~B} ; t=28.0,85 \% \mathrm{~A} / 15 \% \mathrm{~B} ; t=$ $36.0,72 \% \mathrm{~A} / 28 \% \mathrm{~B} ; t=56.0,52 \% \mathrm{~A} / 28 \% \mathrm{~B} / 20 \% \mathrm{C} ; t=70.0$, $1 \% \mathrm{~B} / 98 \% \mathrm{C} / 1 \% \mathrm{D}$; and $t=90.0,80 \% \mathrm{C} / 20 \% \mathrm{D}$. A HewlettPackard pump (model 1050), HP 3396 integrator and the LLSD prepared at the laboratory were used for HPLC.

The previously reported experimental design of LLSD (Stolyhwo et al. 1983, 1984) was substantially modified. In brief, a laser diode (5 $\mathrm{mW}$ at $670 \mathrm{~nm}$; Toshiba, Japan) was used at a controlled working temperature $\left(40 \pm 2{ }^{\circ} \mathrm{C}\right)$ in place of a $\mathrm{He}-\mathrm{Ne}$ laser. A 4-mm wide glass fiber optics (Glass Works, Bialystok, Poland) for transmission of scattered radiation and a photomultiplier (R-372HA, Hammamatsu, Japan) were used together with a chopper-stabilized operational amplifier (CMOS). The essential difference between the ELSD (Varex Corp. USA) and the locally made LLSD (Stolyhwo et al. 1983, 1984, 1985, 1987) consisted of optimizing the LLSD for the lowest possible work temperature at which the eluate from the column was nebulized and the solvents evaporated. That aim was accomplished by restricting the maximum flow rate to the nebulizer to $0.2 \mathrm{~mL} / \mathrm{min}$ (stream splitter with controlled resistance to flow used before the nebulizer). Limitation of the eluate flow reduced the amount of heat (hence nebulizer and drift tube temperature) supplied to the nebulizer for solvent vaporization. Consequently, the geometry and mechanical dimensions of the LLSD were optimized. The reduced eluent flow rate and optimized geometry of the LLSD improved its quantitative characteristics. At present, the LLSD enables detecting methyl laurate $\mathrm{C} 12: 0$ in acetone at the temperature of the nebulizer and drift not exceeding $30^{\circ} \mathrm{C}$.

For the isolation of fractions from the HPLC column, the eluate containing individual peaks or groups of peaks was collected manually into a tapered borosilicate vials (Clear GOLD, Chromacol Ltd., Welwyn Garden, Great Britain) using a stream splitter installed just before the nebulizer of the LLSD. In order to confirm the identification of individually collected FAMEs, their fractions from the HPLC were then re-injected into the GC. Retention times (RT) of separated peaks were compared with those of corresponding standards. Quantitative characteristics of the LLSD detector were reported earlier (Stolyhwo et al. 1984).

\section{Results and Discussion}

Separation of Structural and Geometric Isomers of Fatty Acids from Cold-Pressed Rapeseed Oil

The FAMEs of cold-pressed rapeseed oil may be separated into individual components by capillary GC on the Rtx 2330 column or into classes, according to the number of double bonds and geometrical structure in the FA molecules, with the use of $\mathrm{Ag}^{+} / \mathrm{HPLC}$. The HPLC separation may be conducted on a silver-loaded column using gradient elution and the LLSD. Examples of such separations are shown in Figs. 1a and 2a. The results in Fig. 1a indicate two naturally occurring $c i s$ isomers of $\mathrm{C} 18: 1-9 c$ (oleic acid; $53.1 \%$ ) and $11 \mathrm{c}$, vaccenic acid (3.3\%) in rapeseed oil. Other monounsaturated FAMEs, C16:1, C20:1, and C22:1, are also accompanied by the corresponding cis isomers with double bonds shifted in the hydrocarbon chain by two carbon atoms like in the oleic acid. Separation of the above isomers by GC was accomplished with the use of a very polar stationary phase.

In $\mathrm{Ag}^{+}$HPLC, the interactions of $\pi$ electrons of FAs with silver ions are very strong (Christie et al. 1988). Thus, the silver-loaded column is very selective with respect to the number of double bonds, geometric configuration, and the positions of double bonds in the FA molecules. This is clearly shown in Fig. 2a in which rapeseed FAMEs are separated on a silver-loaded column into saturated, mono, di-, and tri-unsaturated classes of compounds. The elution of FAME classes with increasing numbers of double bonds requires switching the mobile phase composition to much stronger solvents from hexane to methylene chloride, acetone and, finally, to acetonitrile as shown in Fig. 2a. It is essential that column regeneration in HPLC experiments be done carefully with dry solvents according to the manufacturer's specifications. The order of regeneration must be exactly opposite to that used in the original gradient in the volume ratios included in the graphs. The temperature must remain constant to within $\pm 1^{\circ} \mathrm{C}$. While some changes in RT may be observed, the elution pattern remains constant at proper regeneration of the column.

The column is not selective with respect to the chain length of FAs. Small peaks corresponding to C16:1, C20:1, and $\mathrm{C} 22: 1$, just before the $\mathrm{C} 18: 1-9 c$ peak, were separated from the main peak because of different positions of double bonds in the FA molecule with respect to the carboxyl group. Another group of small peaks eluted before the large di- and tri-unsaturated peaks are the positional and/or geometric isomers of di- and tri-unsaturated FAMEs, respectively; these were probably generated during saponification and methylation of FAs. The peaks in Fig. 2a were identified after collecting fractions from HPLC into small tapered glass vials and partial preconcentration followed by re-injection into the GC column.

$\mathrm{Ag}^{+} / \mathrm{HPLC}$ and GC Separation of FA's of Hydrogenated Rapeseed Oil

The separation of FAMEs contained in the industrially hydrogenated rapeseed oil is shown in Fig. 2b. In order 
Fig. 1 a GC separation of FAMEs from cold-pressed rapeseed oil. Identification of FAs: 1 mirystic, C14:0; 2 palmitic, C16:0; 3 stearic, C18:0; 4 oleic, C18:1-9c; 5 vaccenic C18:1-11 cis; 6 linoleic, C18:2-9c,12c; 7 eicosanoic, C20:0; 8 linolenic C18:39,12,15allc; 9 gadoleic, C20:1; 10 behenic, $\mathrm{C} 22: 0 ; 11$ erucic, C22:1. b GC separation of C18:1 trans (fraction III) FAME positional isomers of hydrogenated rapeseed oil collected from $\mathrm{Ag}^{+} / \mathrm{HPLC}$ run (Fig. 2b). Legend: $E A=$ elaidic acid, C18:19t. Proportions (\%) of isomers are shown in brackets. Multiple trans isomers of C20:1 and C22:1 detectable (not indicated). CS denotes change in integrator chart speed. c GC separation of C18:1 cis (fraction IV) FAME positional isomers of hydrogenated rapeseed oil collected from $\mathrm{Ag}^{+} / \mathrm{HPLC}$ run (Fig. 2b) Legend: $O=$ oleic $\mathrm{C} 18: 19 c$. Small quantities of isomers C18:2 (IVa) and C18:3 detectable (not shown). Proportions $(\%)$ of isomers shown in brackets. CS denotes change in integrator chart speed
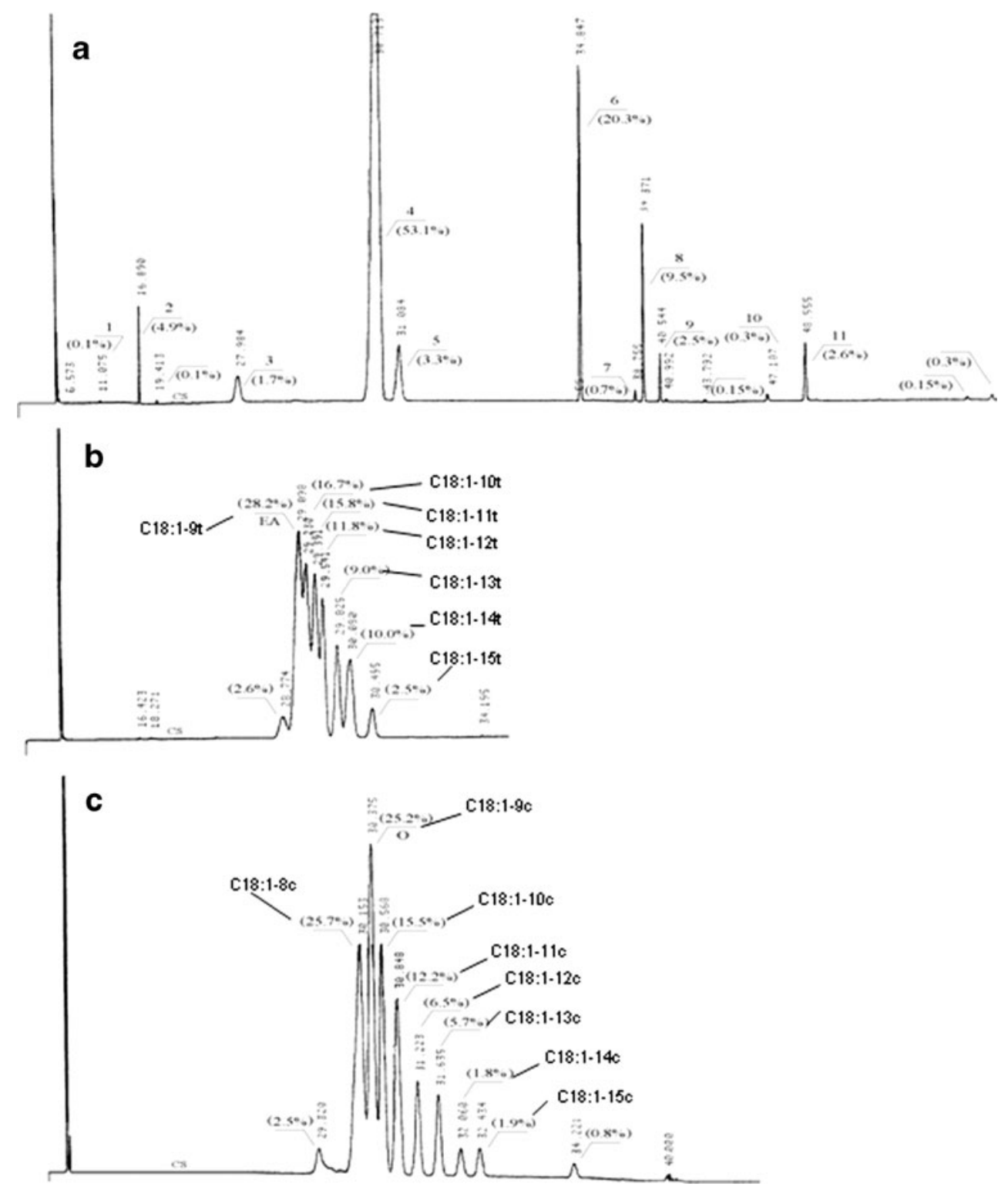

to more clearly show the composition of both the principal and minor components of a sample, various classes of separated analytes were recorded at different attenuations and integrator chart speeds (as marked in Fig. 2b) by the letters AT and CS, respectively. The order of elution depends, as expected, on the interaction of different FAMEs with the packing material. At the beginning, all the saturated FAMEs, fraction I, are eluted irrespectively of the hydrocarbon chain length. The small peak, fraction II, just after the saturated FAMEs, is related to the saturated branched chain FAs which may be formed in small quantities during the process of industrial catalytic hydrogenation of rapeseed oil. Also, impurities adsorbed from hexane during the regeneration of the column may be present in this peak. The peak marked ATF is such an impurity from hexane as confirmed by a blank run (not shown). The group of peaks marked as fraction III corresponds to monoenoic FAMEs, all of trans configuration. Individual peaks in this group correspond to structural (positional) monoenoic trans isomers. This was also confirmed by GC as discussed below. The next group of peaks (fraction IV) corresponds to structural (positional) monoenoic isomers, all of cis configuration. The later elution of $c i s$ isomers with respect to trans isomers is related to a stronger interaction of silver ions with $\pi$ electrons in cis double bonds than in the trans ones. The subsequent groups of peaks (fractions V, VI, and VII), correspond to the structural (positional) isomers of dienoic C18:2 (linoleic acid) having trans-trans, cis-trans, and cis-cis configurations, respectively. The monoenoic trans isomers are known to quantitatively dominate in hydrogenated fats, therefore the GC analysis of those fractions was not presented. 
Fig. $2 \mathrm{Ag}^{+} / \mathrm{HPLC}$ separation of FAMEs. a Cold-pressed rapeseed oil retention times (RT) shown for main peaks. ATF artifacts (from hexane used). b Industrially hydrogenated rapeseed oil. Identification of FAME fractions: $I$ saturated (all chain lengths); II saturated branched chain + ATF; III monoenoic trans structural (positional) isomers; $I V$ monoenoic cis positional isomers; $V$ dienoic trans-trans positional isomers; $V I$ dienoic trans-cis positional isomers; and VII dienoic cis-cis positional isomers. ATT denotes change in attenuation and $C S$ denotes change in integrator chart speed

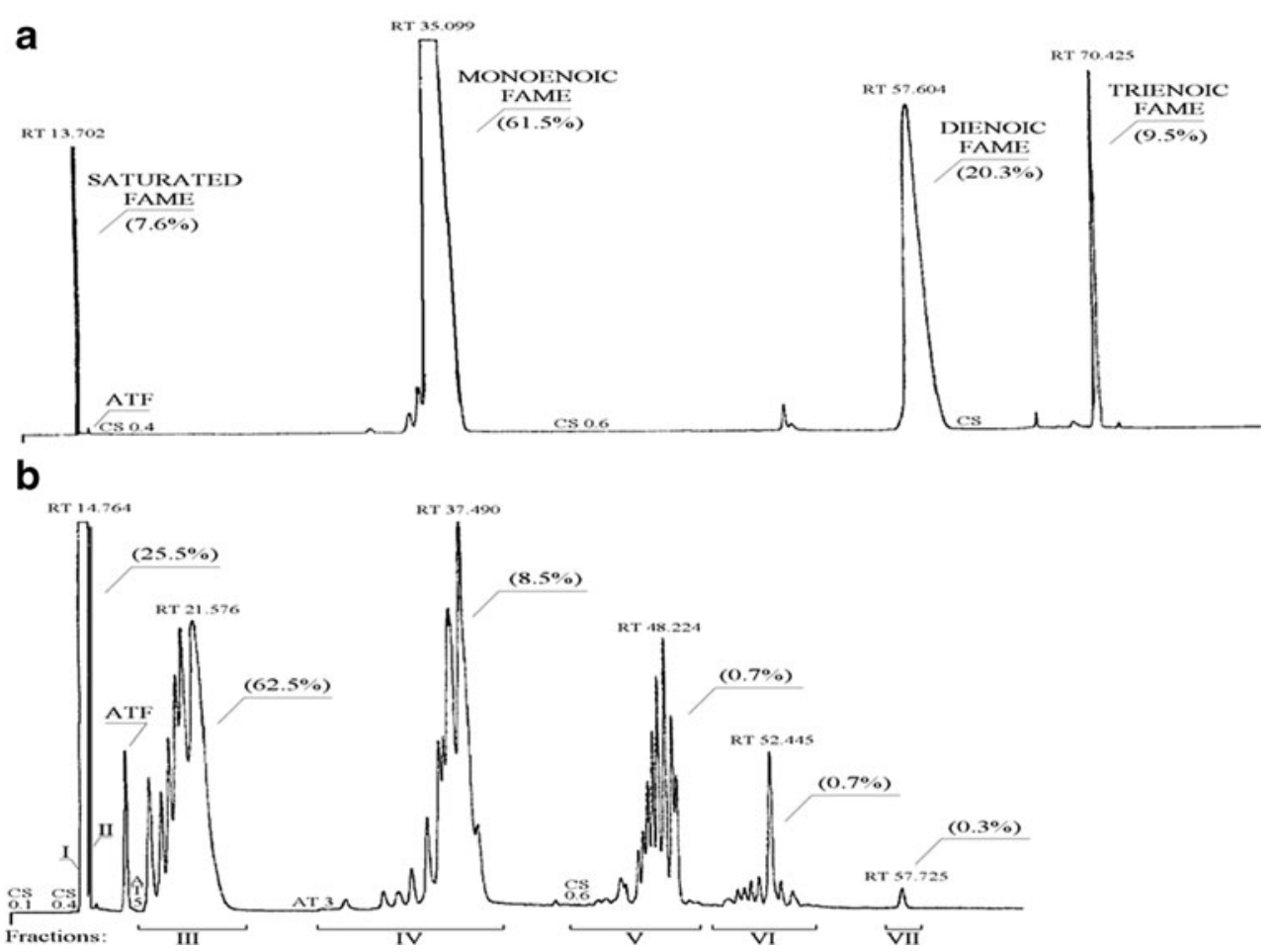

\section{GC Analysis of Separated Fractions}

A small split system was installed at the outlet of the $\mathrm{Ag}^{+}$/ HPLC column just before the nebulizer of the LLSD in order to collect the separated fractions as marked in Fig. 2. After evaporation of about $90 \%$ of the collected mobile phase, the fractions were analyzed by GC. The chromatograms of fractions III and IV are shown in Fig. 1b and c, respectively. As shown in Fig. 1b, the trans fraction of monoenoic C18:1 FAME can be separated into eight structural (positional) isomers. The elaidic acid, C18:1-9t, marked as EA in the graph constitutes about $28 \%$ of the $\mathrm{C} 18: 1$ trans isomers. The relative concentration (in brackets) of individual trans isomers can be considered a specific "profile" of the hydrogenated fat. Comparison of the profiles of monoenoic trans and cis isomers of different fats may be useful in determining the origin of a specific hydrogenated fat or mixture of fats. Besides C18:1, other monoenoic FAMEs, such as multiple peaks corresponding to C20:1 and C22:1 trans isomers, can be found in the chromatogram (not shown). Fraction IV, the cis isomers of monoenoic FAMEs under the described experimental conditions, can be separated into nine positional (structural) isomers (Fig. 1c) in which oleic acid marked as $\mathrm{O}$ constitutes about $25 \%$ of the total $\mathrm{C} 18: 1 \mathrm{cis}$ isomers (last peak RT 34.221 remains unidentified). The relative proportions (in brackets) between individual isomers are shown in the graph. Quantitative data can be used for constructing histograms in order to facilitate a comparison of profiles of monoenoic fractions of $\mathrm{C} 18: 1$ isomers of different fats and their blends with hydrogenated fats. Positional isomers of $\mathrm{C} 18: 1$ other than $9 c$ (oleic) or $11 c$ (vaccenic) do not naturally occur in commonly used edible plant oils. Thus, the presence of other positional isomers should indicate an admixture of hydrogenated fats into the sample in question. An approximate quantitation of added hydrogenated fat (if available) could be made on that basis. Small amounts of other positional monoenoic cis isomers, namely C20:1 and C22:1 (not shown), can also be detected in Fraction IV.

Some comments related to the separation of cis-trans isomers of FAMEs on the basis of the presented data ought to be made here. The separation by GC of positional isomers within the trans fraction is slightly less effective than that of positional isomers within the cis fraction. In general, when compared with the GC separation of rapeseed oil FAMEs (Fig. 1a), the trans fraction is eluted before the cis fraction of C18:1 isomers on the Rtx 2330 column. Some trans isomers are overlapping, however, with cis isomers. Branched-chain C18:0 (saturated) FAMEs (not shown) are also eluted in the same region of retention times. These are the reasons why GC itself, even with the use of very efficient long columns loaded with highly polar stationary phases, is not sufficient to characterize the composition of hydrogenated fats. The same conclusion, but on the basis of different data, was drawn by Ratnayake and Beare-Rogers (1990).

One approach used to identify the elution positions of various geometric isomers involved isomerizing the oils with nitrous oxide. The results are shown in the chromatogram of the products (Fig. 3). Each of the major groups identified in the hydrogenated oil (Fig. 2b) are present in the FAME 
Fig. $3 \mathrm{Ag}^{+} / \mathrm{HPLC}$ separation of FAMEs from rapeseed oil isomerized by nitrous oxide; N.I.- unidentified

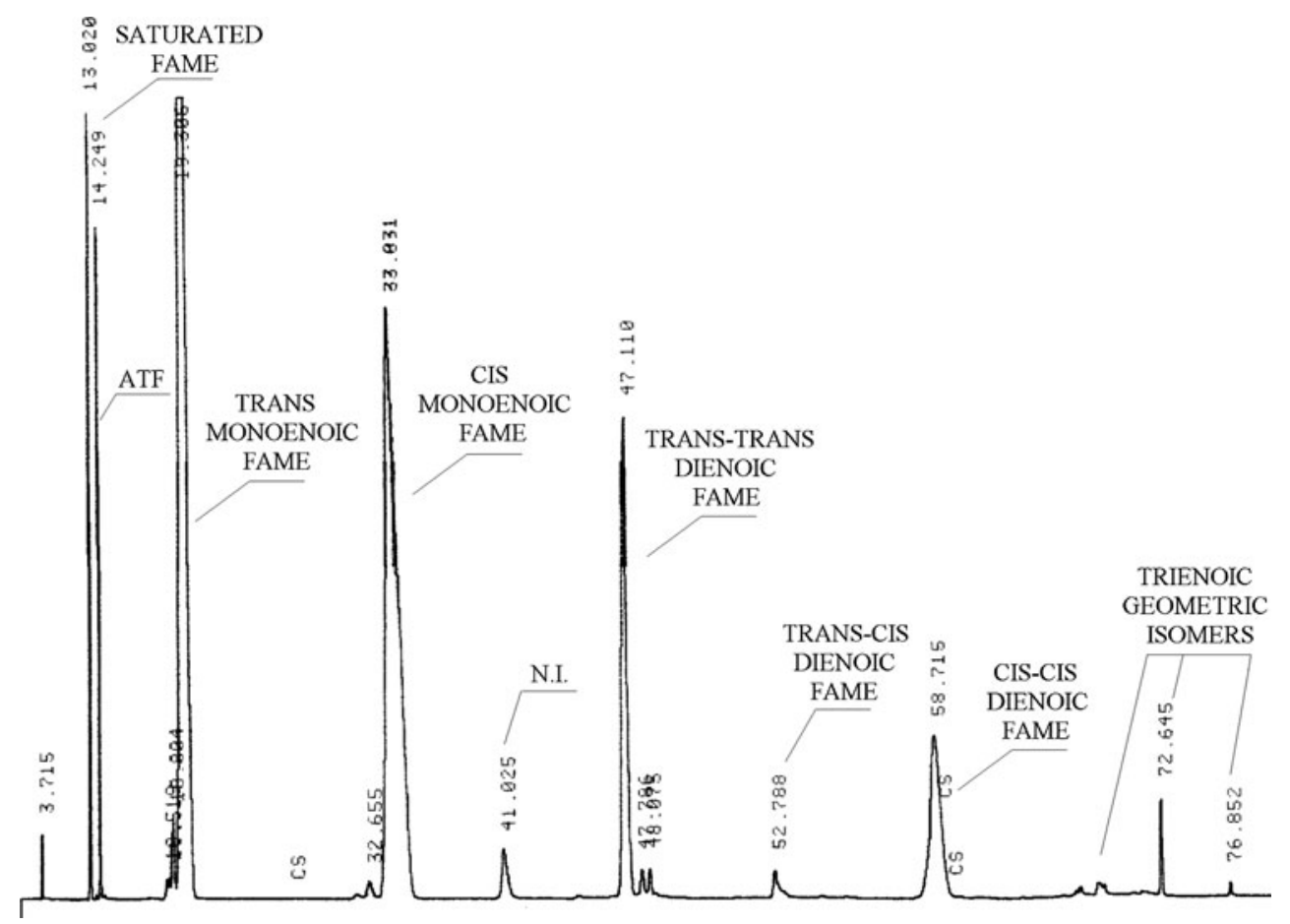

chromatogram of the nitrous oxide-treated oil. However, there are no positional isomers in this mixture (Fig. 2a) which is clearly different from the material obtained by catalytic hydrogenation. Therefore, the industrial treatment process can be considered to have even more drastic effects on the virgin oil than the nitrous oxide treatment.

The use of $\mathrm{Ag}^{+} / \mathrm{HPLC} / \mathrm{LLSD}$ followed by GC of the collected fractions at present seems to be a practical solution to the question of analysis of the composition of hydrogenated fats. The procedure may also be useful for determining the selectivity of catalysts used, process monitoring of industrial hydrogenation of oils, quality control of hydrogenated fats, etc. The composition of fractions V and VI, C18:2, is very complex (not shown). Over 20 peaks were separated by GC on the Rtx 2330 column. The structures of the individual peaks were not identified.

\section{Quantitative Assessment of $\mathrm{Ag}^{+} / \mathrm{HPLC} / \mathrm{LLSD}$ Chromatograms}

As reported earlier (Stolyhwo et al. 1983, 1984, 1985, 1987), the LLSD signal was not linearly related to the mass or concentration of the analyte. After the technical improvements of the LLSD described above, the following simplified relationship may be used for preliminary assessment of the content of trans FA in analyzed fats:

$S \approx k c^{x}$,

where $S$ is the signal of the LLSD expressed as the number of counts of the integrator, $k$ is the response factor which may be assumed equal to one (Stolyhwo et al. 1983), $c$ is the concentration of the analyte, and the exponent $x$ is slightly dependent on the composition of the mobile phase. Consequently, in case of gradient elution, small fluctuations in $x$ may be expected. For preliminary quantitation, a mean value of $x=1.12$ can be used. In the "internal normalization" method, peak areas (in counts) as printed by the integrator, should be corrected according to the above simplified equation. If more precise quantitative data are required, the calibration of the system must be conducted.

An example of quantitation of HPLC separations on the basis of the above equation is shown in Table 1 in which data from the chromatograms shown in Figs. 1a (GC) and 2a (HPLC, five consecutive runs) are compared. As shown in Table 1, the $\mathrm{Ag}^{+} /$HPLC/LLSD technique enabled

Table 1 Quantitation of FAME components in rapeseed oil by capillary GC and HPLC/LLSD

\begin{tabular}{|c|c|c|c|c|}
\hline \multirow[t]{2}{*}{ Rapeseed oil FAMEs } & \multicolumn{2}{|c|}{$\begin{array}{l}\text { Cold-pressed oil } \\
\text { FAME }(\%)\end{array}$} & \multicolumn{2}{|c|}{$\begin{array}{l}\text { Hydrogenated oil } \\
\text { FAME }(\%)\end{array}$} \\
\hline & $\mathrm{GC}$ & $\begin{array}{l}\text { HPLC/ } \\
\text { LLSD }\end{array}$ & $\mathrm{GC}$ & $\begin{array}{l}\text { HPLC/ } \\
\text { LLSD }\end{array}$ \\
\hline Saturated & $7.8 \pm 1.3$ & $7.6 \pm 0.6$ & $23.8 \pm 2.4$ & $25.5 \pm 2.7$ \\
\hline trans monounsaturated & ND & $\mathrm{ND}$ & $58.8 \pm 4.1$ & $62.5 \pm 2.4$ \\
\hline cis monounsaturated & $62.1 \pm 1.7$ & $61.5 \pm 2.0$ & $13.9 \pm 1.2$ & $8.5 \pm 0.9$ \\
\hline Sum of monounsaturated & $62.1 \pm 1.7$ & $61.5 \pm 2.0$ & $72.7 \pm 5.2$ & $71.0 \pm 4.2$ \\
\hline Diunsaturated & $20.4 \pm 1.8$ & $20.3 \pm 1.5$ & ND & ND \\
\hline Triunsaturated & $9.4 \pm 1.5$ & $9.5 \pm 1.0$ & ND & ND \\
\hline $\begin{array}{l}\text { Other unidentified } \\
\text { isomers }\end{array}$ & ND & $\mathrm{ND}$ & $4.4 \pm 0.7$ & $2.7 \pm 0.3$ \\
\hline
\end{tabular}


demonstrating that in spite of the desired selective saturation of the double bonds of linolenic (C18:3) and linoleic (C18:2) acids, the principal process of industrial hydrogenation of oil results in the formation of isomers of FAs, structural (positional) and geometric (cis/trans), the positional isomerization being the prevailing process. Both cis and trans isomers are positionally isomerized as shown in Fig. 2b. The second process is geometric isomerization. In this case, $62.6 \%$ of the total FAs (about $88 \%$ of C18:1 FA) is converted into trans isomers. Assuming that the peak RT 37.490 corresponds to oleic acid C18:1-9c, it is possible to estimate from the integrated chromatogram (not shown) that only $2.3 \%$ of the monounsaturated FA left in hydrogenated oil is neither structurally nor geometrically isomerized. Differences in the contents of trans and cis isomers, resulting from the use of GC or HPLC (Table 1), were due to the earlier reported overlapping (Delmonte and Rader 2007, AOCS Ce 1h-05).

Taking into consideration the above analytical results indicating considerable changes of FA structure of hydrogenated fats, as well as the randomization of triacylglycerols which takes places during hydrogenation, the utilization of these fats as regular dietary components may indeed be regarded as controversial. Some examples of analyses by $\mathrm{Ag}^{+} /$ HPLC/LLSD of products containing hydrogenated fats are presented below.

\section{Determination of Hydrogenated Fats in Food Products}

Soft Margarine The $\mathrm{Ag}^{+} / \mathrm{HPLC} / \mathrm{LLSD}$ chromatogram of a soft margarine is shown in Fig. 4. Five FAME classes were discerned: I, saturated FA (18.3\%); II, monoenoic trans positional isomers (10.9\%); III, monoenoic cis FA
(33.3\%); IV, dienoic cis-cis FA (32.4\%); and V, trienoic all cis FA (3.8\%). The addition of hydrogenated fat to the blend of natural fats is clearly visible. Structural (positional) cis isomers in relatively low concentrations are contained in the monoenoic FA peak and could be identified by collecting the peak from the HPLC-GC analysis as shown above for hydrogenated fat.

Margarine (Hard) for Baking Fats used for baking should have an increased stability against oxidation at elevated temperatures. At present, this requirement is partially achieved by selective hydrogenation mainly of the linolenic (C18:3) and linoleic (C18:2) acids present in plant oils, possibly without affecting monoenoic acids, more resistant to oxidation. However, the undesired side-effects of the process are structural and geometric isomerization of the monoenoic FAs. Such "partially hydrogenated" fats are contained in bakery products in quite large quantities. An example of $\mathrm{Ag}^{+} / \mathrm{HPLC} / \mathrm{LLSD}$ analysis of the composition of fat used for baking is shown in Fig. 5. Fraction II, monoenoic trans positional isomers, constitutes $39.4 \%$ of the total FAs present in the product. Also, various isomers of C18:2 (fractions IV and V) are detectable. After collection and analysis of fractions IV and V, 18 peaks (not shown) corresponding to $\mathrm{C} 18: 2$ isomers are detectable. Such isomers cannot be considered "essential" FAs, neither they are naturally occurring in the commercial edible plant oils.

French Fries Fat from French fries was extracted with methylene chloride. The extracted fat $(22 \mathrm{~g} / 100 \mathrm{~g}$ of dried product) was converted to FAMEs and analyzed by $\mathrm{Ag}^{+} /$ HPLC/LLSD. The resulting chromatogram is shown in Fig. 6. The following fractions were separated: Fraction I,
Fig. $4 \mathrm{Ag}^{+} / \mathrm{HPLC}$ separation of FAMEs from soft margarine. Identification of FAME fractions: $I$ saturated; $I I$ monoenoic trans structural isomers; III monoenoic cis isomers; $I V$ dienoic (mainly linolenic acid); and $V$ trienoic (mainly $\mathrm{C} 18: 39,12,15$ all cis). $A T F=$ artifact from hexane. Proportions (\%) shown in brackets

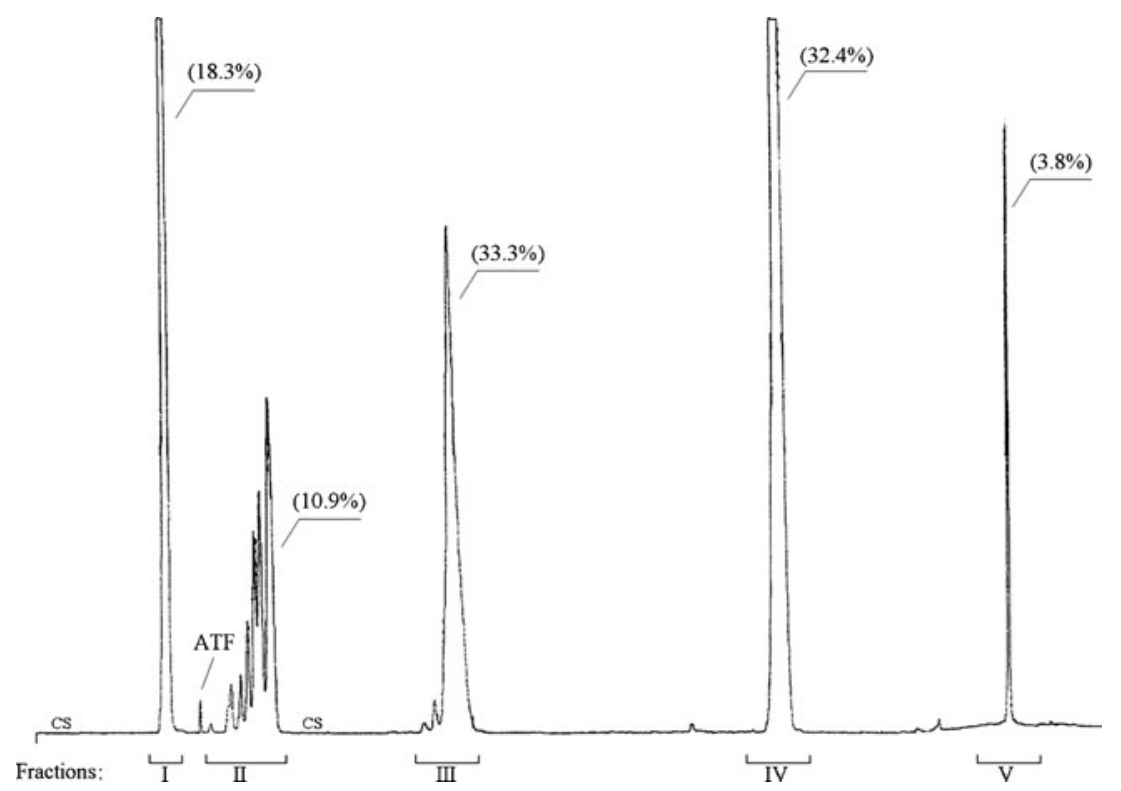


Fig. $5 \mathrm{Ag}^{+} / \mathrm{HPLC}$ separation of FAMEs from hard margarine for baking FAME fractions: $I$, $I I$, and III as in Fig. 4; IV, dienoic cis-trans isomers; $V$ dienoic cis-cis (linoleic C18:2 9,12 cis-cis); and $V I$ trienoic acid C18:3. Proportions (\%) shown in brackets

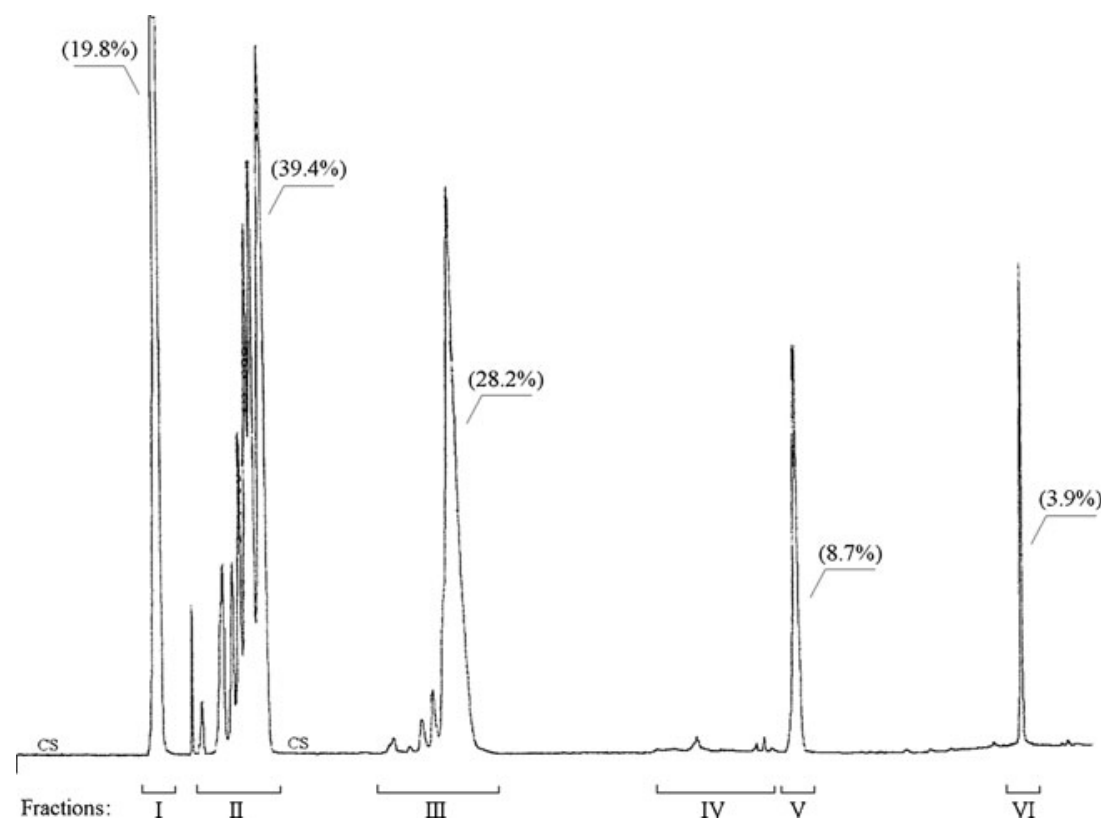

saturated species, amounting to $68 \%$ of the total FAMEs; fraction II, monoenoic trans positional isomers 23.2; and fraction III, monoenoic cis positional isomers $7.7 \%$. It becomes evident from the above data that French fries, as they are actually prepared, are a considerable source of undesired saturated and isomeric FAs.

Structural and Geometric Isomers of Fatty Acids in Human Milk

The addition of hydrogenated fats into food products like margarine, spreads, bakery goods, cooking fats, emulsifiers, creamers, etc., is likely to have an effect on the composition of fatty acids present in human body. The usefulness of the combined $\mathrm{Ag}^{+} / \mathrm{HPLC} / \mathrm{LLSD}$ and GC techniques for the determination of hydrogenated fats in structural lipids may be demonstrated by the analysis of FA isomers present in human milk.

The use of GC enables obtaining partial separation of trans isomers from other FAs present in the sample as shown in Fig. 7. However, identification and quantitation of all FA isomers is not straightforward. For comparison, an $\mathrm{Ag}^{+} / \mathrm{HPLC}$ chromatogram of the same sample is shown in Fig. 8; it differs from GC results as the sample was separated
Fig. $6 \mathrm{Ag}^{+} / \mathrm{HPLC}$ separation of fat extracted from French fries fractions: $I$ saturated; $I I$ monoenoic trans positional isomers; III monoenoic cis positional isomers same as in Fig. 3. Proportions (\%) shown in brackets

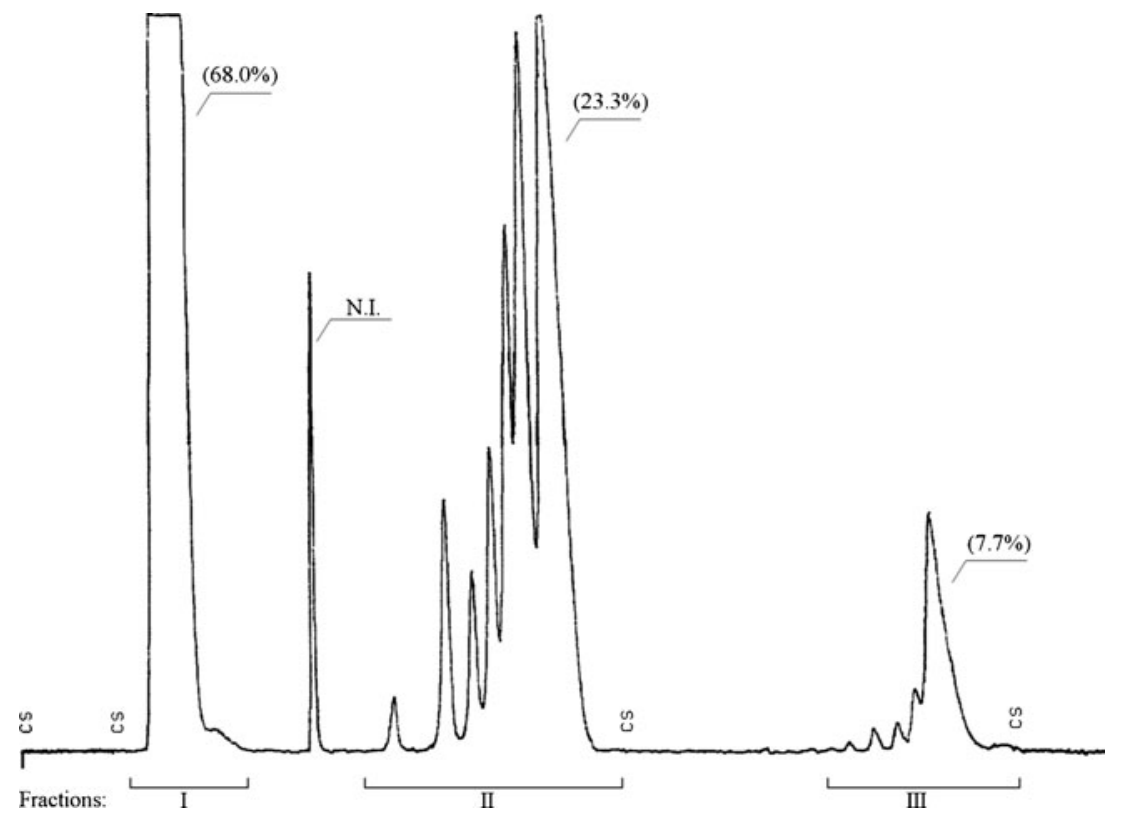


Fig. 7 GC separation of human milk fat FAMEs Identification of main FAME components: 1 C10:0; 2 C12:0; 3 C14:0; 4 C16:0; 5 C16:1 (some adjacent peaks are positional isomers of C16:1); 6 C18:0; 7 C18:1 (multiple positional trans isomers); $8 \mathrm{C} 18: 1-9 \mathrm{cis}$; 9 C18:1-11cis; 10 C18:2 cis-cis; 11 C20:0; 12 C18:3-9,12,15 all cis; and $13 \mathrm{C} 20: 1$. CS denotes change in integrator chart speed

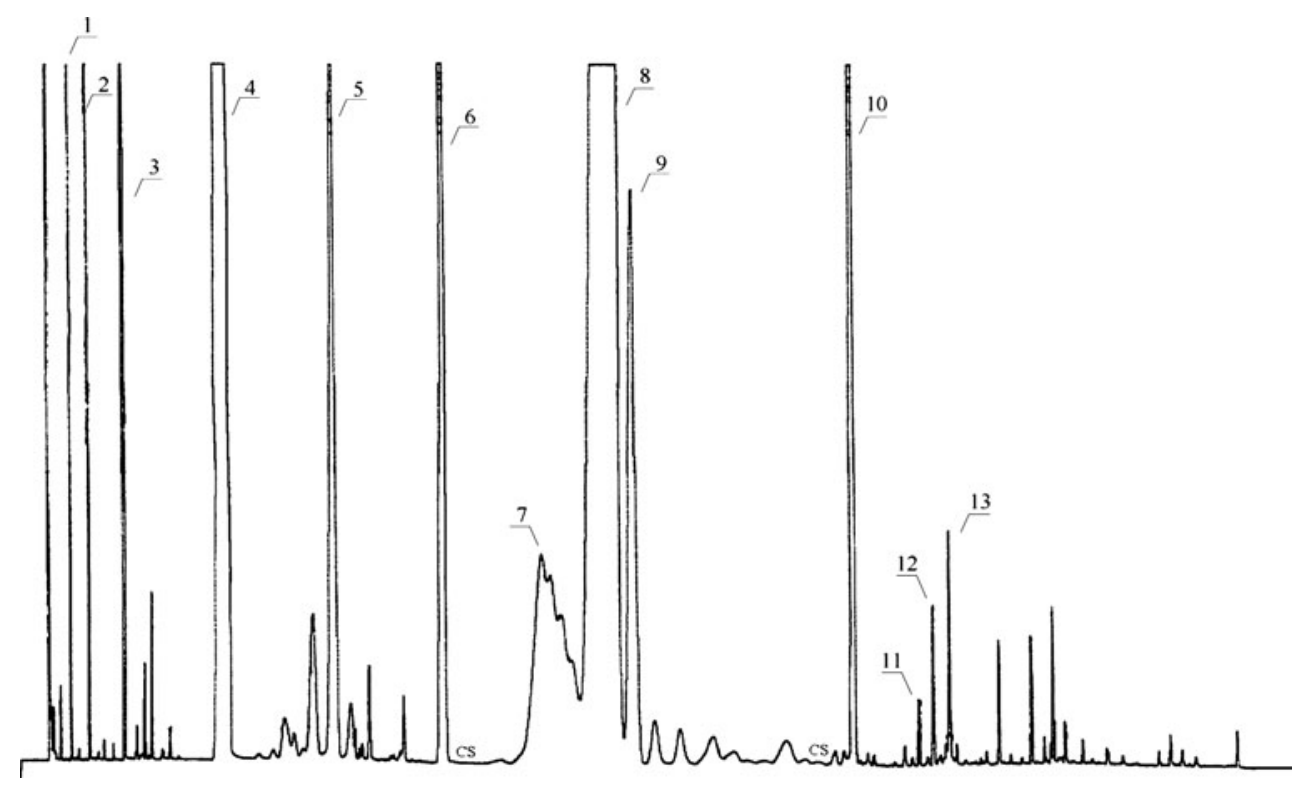

into classes of FAs with different degrees of unsaturation and geometric structure. Individual fractions of FAMEs, as marked in Fig. 8, were identified as follows: I, saturated (46.7\%); II, monoenoic trans positional isomers (3.1\%); III, monoenoic cis FAs (36.7\%); IVa, dienoic cis-trans isomers (1.3\%); IVb, dienoic cis-cis isomers (9.2\%); and $\mathrm{Va}, \mathrm{b}, \mathrm{c}, \mathrm{d}, \mathrm{e}$, isomers of tri- and higher unsaturated FAs $(1.1 \%)$. As indicated in Fig. 8, each fraction was collected during the HPLC run and, after concentration of given fraction, re-analyzed by GC. The GC results of monoenoic trans and cis fractions are shown in Fig. 9a and b, respectively. As shown in Fig. 9a, fraction II (trans) can be separated into eight structural C18:1 isomers and resembles the profile of hydrogenated fat (Fig. 1b). It may thus be concluded that dietary structural and geometric FA isomers from hydrogenated fats passed unchanged into the milk. Such observation was confirmed by the work done by Mueller et al. (2010). Figure 10 shows the trans fraction of monoenoic FAs isolated from late summer bovine milk when the content $(4.7 \%)$ of trans monoenoic isomers was found highest compared with
Fig. $8 \mathrm{Ag}^{+} / \mathrm{HPLC}$ separation of human milk fat FAMEs Conditions of separation same as in Fig. 2a. Proportions (\%) shown in brackets

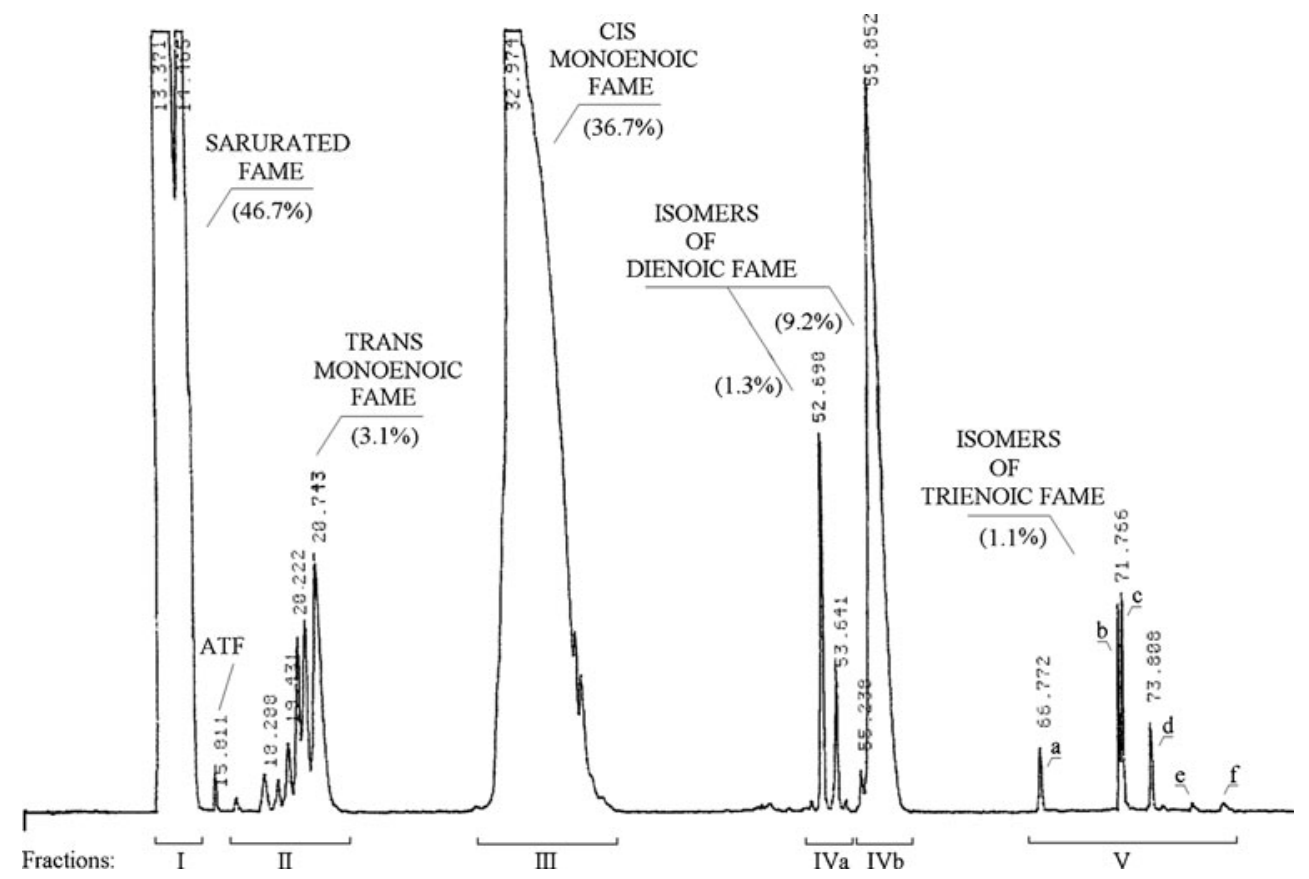


Fig. 9 GC separation of trans and $c i s$ isomers from human milk fat. a trans isomers of fraction II (Fig. 8). Proportions (\%) shown in brackets. CS denotes change in integrator chart speed. $\mathbf{b}$ cis isomers of fraction III (Fig. 8). Legend: $O=$ oleic acid and $V A=$ vaccenic acid. Proportions (\%) shown in brackets. $C S$ denotes change in integrator time scale
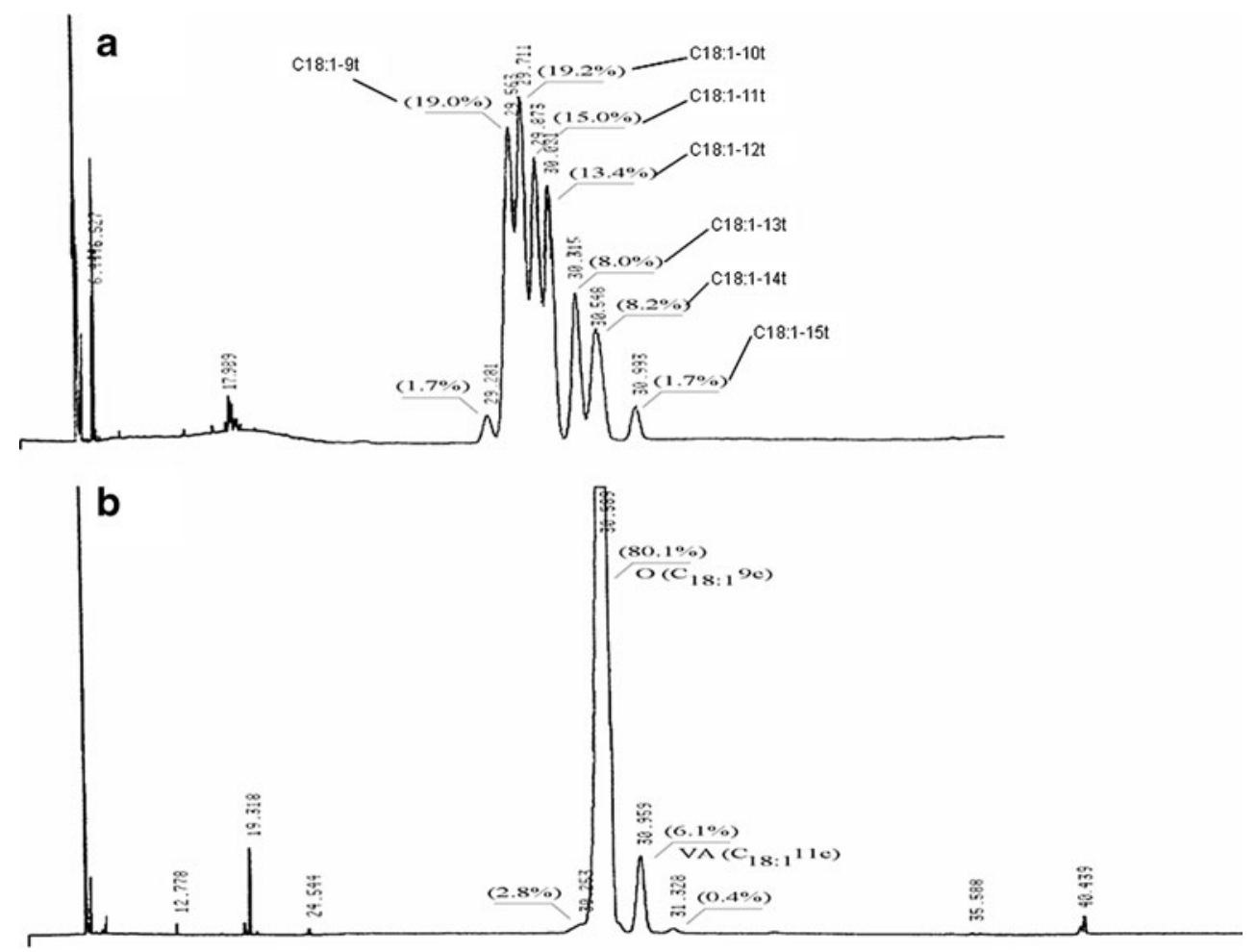

other seasons of the year. As seen in Fig. 10, the trans fraction was separated partially into seven geometric isomers. The main component (74 \% of the C18:1 trans isomers) was identified as trans vaccenic acid (C18:1-
$11 t)$; generally, this is in agreement with other reports (Wolff 1995; Laakso and Kallio 1993; Precht and Molkentin 1994). The profiles of C18:1 trans isomers of bovine milk and of human milk are dissimilar, although the
Fig. 10 GC separation of C18:1 trans isomer fraction isolated from bovine milk. Legend: $t V A=$ trans vaccenic acid. Proportions (\%) shown in brackets. CS denotes change in integrator chart speed

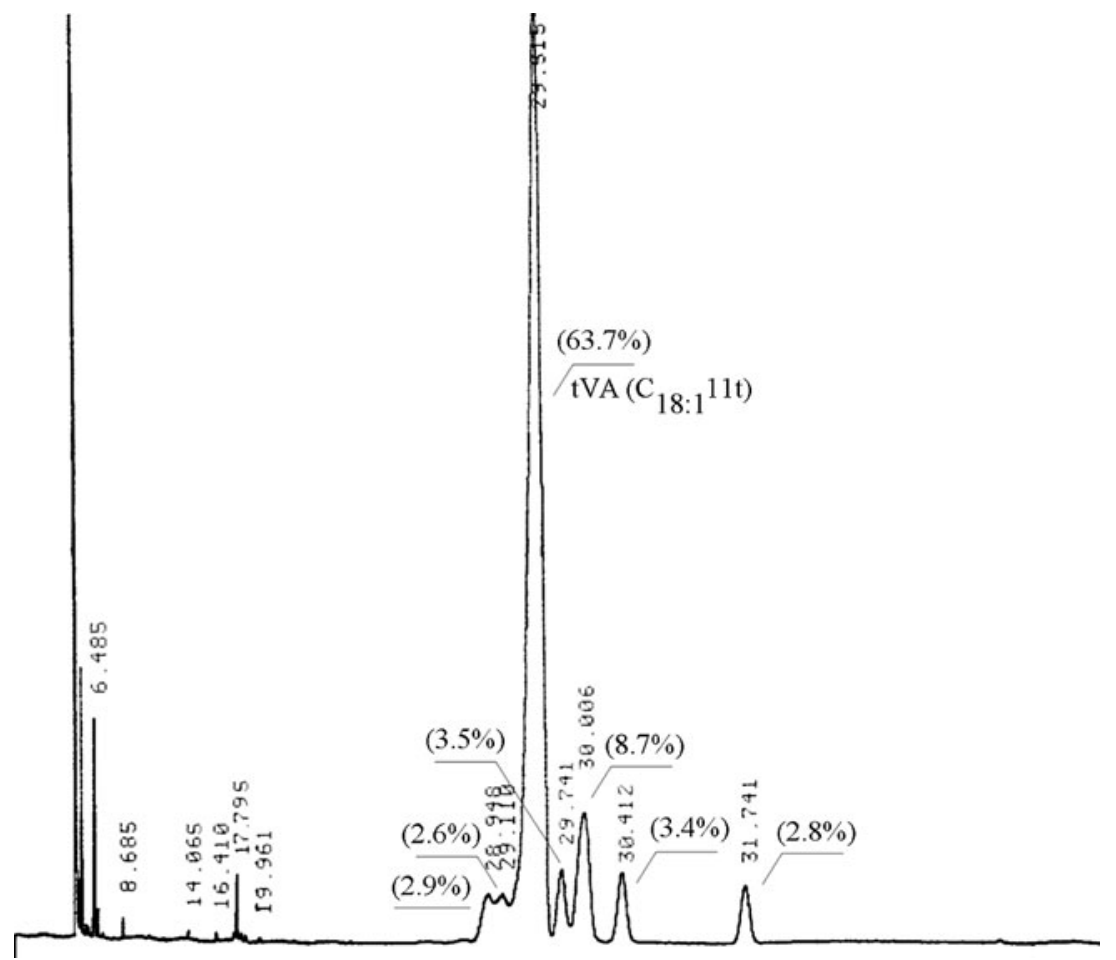


latter may be slightly affected by $\mathrm{C} 18: 1-11 t$ from dairy products contained in woman's diet. Beside C18:1, other monoenoic trans FA isomers were detected in the trans fraction of human milk as shown in the chromatogram. As can be seen in Fig. 9b, in contrast to the trans fraction, the C18:1 cis fraction of FAs in human milk contains virtually only two structural isomers: oleic C18:1-9c, and vaccenic $\mathrm{C} 18: 1-11 c$, which are the only monoenoic FAs naturally occurring in plant oils. Other structural cis isomers present in large quantities in hydrogenated fats (Fig. 1c), and hence in dietary fats, occur in human milk only in negligible quantities. It is interesting that a similar cis fraction of $\mathrm{C} 18: 1$ in bovine milk fat also contains exclusively oleic and vaccenic acids. It was not possible to detect other cis isomers in bovine milk even when considerable quantities of a sample were injected into the GC.

It was not the scope of this paper to explain what happened physiologically to the positional C18:1 cis isomers other than oleic and vaccenic acids present in hydrogenated fat (Fig. 1c) of a typical diet. However, the analytical approach presented here may prove helpful to others in explaining this phenomenon.

\section{Summary and Conclusions}

The use of $\mathrm{Ag}^{+} / \mathrm{HPLC} / \mathrm{LLSD}$ followed by GC of the collected fractions at present seems to be a practical solution to the question of analysis of the composition of hydrogenated fats. The procedure may also be useful for determining the selectivity of catalysts used for hydrogenation, process monitoring of industrial hydrogenation, quality control of hydrogenated fats, etc.

The FAMEs of hydrogenated fats may be separated into classes according to the degree of unsaturation or geometric (cis/trans) isomerization. Partial separation of individual components within each class is also possible. This can be done by use of $\mathrm{Ag}^{+}$-loaded HPLC column, gradient elution, and an adequate detection system. So far, only the light scattering detection-based principle, such as an LLSD, can be used for this purpose. Individual fractions may be collected and re-analyzed in a second dimension, namely HRGC. A profile of the composition of the trans or cis fractions can be used for identification of the presence of hydrogenated fats in food products. The method may also be very useful for determining the selectivity of catalysts used in hydrogenation as well as for quality control of hydrogenated fats.

The comparison of profiles of trans FAMEs showed that hydrogenated fats contained in human milk $(3.1 \%)$ originate mainly from dietary sources. trans FAs from bovine milk have an entirely different composition. It remains unclear why only two $(\mathrm{C} 18: 1-9 c$ and $\mathrm{C} 18: 1-11 c)$ of the nine positional cis isomers present in hydrogenated dietary fats are found in human milk while all eight positional trans isomers, found in those food products, could be detected in human milk.

Open Access This article is distributed under the terms of the Creative Commons Attribution License which permits any use, distribution, and reproduction in any medium, provided the original author(s) and the source are credited.

\section{References}

American Oil Chemists Society (2005) Method Ce 1h-05, in official methods and recommended practices AOCS, 5th edn. AOCS Champagin

Applewhite TH (1993) Nutr Rev 51:344-345

Association of Official Analytical Chemists International (AOAC) (1990a) Official method 985.21. In: Helrich K (ed) Official methods of analysis of AOAC International, 15th edn. Association of Official Analytical Chemists, Arlington

Association of Official Analytical Chemists International (AOAC) (1990b) Official method 965.34 In: Helrich K (ed) 15th Ed. 1990, (Revised 1997 AOCS-AOAC Method)

Association of Official Analytical Chemists International (AOAC) (1990c) Official method 905.02. In: Helrich K (ed) Official methods of analysis of AOAC International, 15th edn. Association of Official Analytical Chemists, Arlington

Association of Official Analytical Chemists International (AOAC) (1990d) Official method 969.33. In: Helrich K (ed) Official methods of analysis of AOAC International, 15th edn. Association of Official Analytical Chemists, Arlington

Association of Official Analytical Chemists International (AOAC) (2005) Official method 996.06. In: Horowitz W (ed) Official methods of analysis of AOAC International, 18th edn. Association of Official Analytical Chemists, Gaithersburg

American Oil Chemists Society (AOCS) (2009) Recommended Practice Ce $1 \mathrm{~g}-96$

CFIA. Canadian Food Inspection Agency (2008). B.01.513 FDR section 7.18. Guide to food labeling and advertising. Trans fatty acid claims

Chen ZY, Pelletier G, Hollywood P, Ratnayake WMN (1995) Lipids $30: 15-21$

Christie WW (1987) J High Resolut Chromat 10:148-150

Christie WW (1988) J Chromatogr A 454:273-284

Christie WW (1991) Rev Franc Corps Grass 38:155-160

Christie WW, Breckenridge GHG (1989) J Chromatogr 469:261-269

Christie WW, Brechany EY, Stefanow K (1988) Chem Phys Lipids 46:127-135

Craig-Schmidt MC, Weete JD, Faircloth SA, Wickwire MA, Livant EJ (1984) Am J Clin Nutr 39:778-786

Delmonte P, Rader JI (2007) Anal Bioanal Chem 389:77-85

Destaillats F, Golay PA, Joffre F, De Wispelaere, Hug B, Giuffrida F, Fauconnot L, Dionisi F (2007) J Chromatogr A 1145:222-228

Golay PA, Dionisi F, Hug B, Giuffrida F, Destaillats F (2006) Food Chem 101:1115-1120

Handa Ch, Goomer S, Siddhu A (2010) Int J Food Sci Tech 45:1002-1008

Haumann BF (1994) Inform 6:668-678

Juanéda P (2002) J Chromatogr A 954:285-289

Judd JT, Clevidence BA, Muesing RA, Wittes J, Sunkin ME, Podczasy JJ (1994) Am J Clin Nutr 59:861-868

Koletzko B, Mrotzek M, Bremmer HJ (1988) Am J Clin Nutr 47:954-959

Kummerow FA, Zhou Q, Mahfouz MM, Smiricky MR, Grieshop CM, Schaeffer DJ (2004) Life Sci 74:2707-2723

Laakso P, Kallio HJ (1993) Am Oil Chem Soc 70:1161-1171

Larqué E, Zamora S, Gil A (2001) Early Hum Dev Suppl 65:S31-S41

Lichtenstein AH (1993) Nutr Rev 51:340-343 
Lopez-Garcia E, Schulze MB, Meigs JB, Manson JE, Rifai N, Stampfer MJ, Willett WC, Hu FB (2005) J Nutr 135:562-566

Mensink RP, Katan MB (1990) New Engl J Med 323:439-445

Mueller A, thijs C, Rist L, Simões-Wüst AO, Huber M, Steinhart H (2010) Lipids 45:245-251

Patterson HBW (2009) Hydrogenation of fats and oils: theory and practice. AOCS, Illinois

Phillips KM, Ruggio DM, Amanna KR (2010) Food Anal Methods 3:277-294. doi:10.1007/s12161-010-9123-y

Picciano MF, Perkins EG (1977) Lipids 12:407-408

Precht D, Molkentin J (1994) Kieler Milchwirtschaftliche Forschungsberichte 46:249-261

Ratnayake WMN, Beare-Rogers JL (1990) J Chromatogr Sci 28:633-639
Stefanow K, Konaklieva KM, Brechany EY, Christie WW (1988) Phytochemistry 27:3495-3502

Stolyhwo A, Colin H, Guiochon G (1983) J Chromatogr 265:1-18

Stolyhwo A, Colin H, Martin M, Guiochon G (1984) J Chromatogr 288:253-275

Stolyhwo A, Colin H, Guiochon G (1985) Anal Chem 57:1342-1354

Stolyhwo A, Martin M, Guiochon G (1987) J Liq Chromatog RT 10:1237-1253

USFDA, Food and Drug Administration (2010). 21CFR101.9. Nutrition labeling of food

Willet WC, Stampfer MJ, Manson JE, Golditz GA, Speizer FE, Rosner BA, Hennekens CH (1993) Lancet 341:581-585. doi:10.1016/ 0140-6736(93)90350-P

Wolff RL (1995) J Am Oil Chem Soc 72:259-272 